\title{
BOUNDARY VALUE PROBLEMS FOR SECOND ORDER NONHOMOGENEOUS DIFFERENTIAL SYSTEMS
}

\author{
S. C. TEFTELLER
}

ABSTRACT. This paper is a study of second order nonhomogeneous differential systems involving a parameter with boundary conditions specified at two points. By means of a polar coordinate transformation for this system, the existence of eigenvalues is established. The results of this study extend those of Max Mason in that selfadjointness of the problem is not necessary to insure a solution.

1. Introduction. The study of two-point boundary problems for second order homogeneous differential equations has a rich history dating back to the work of Sturm and Liouville. The study of such problems for second order differential systems by means of a polar coordinate transformation appears to have begun with the fundamental work of W. M. Whyburn [7], [8], [9] who credits H. J. Ettlinger with suggesting this method. Whyburn's work has recently been extended to more general two-point boundary problems by G. J. Etgen [2], [3].

This paper considers the second order nonhomogeneous differential system

$$
y^{\prime}=k(x, \lambda) z, \quad z^{\prime}=g(x, \lambda) y+f(x, \lambda),
$$

together with the associated homogeneous system

$$
u^{\prime}=k(x, \lambda) v, \quad v^{\prime}=g(x, \lambda) u .
$$

The coefficient functions $k(x, \lambda), g(x, \lambda)$, and the torcing function $f(x, \lambda)$ are real-valued functions on $X: a \leq x \leq b, L: \lambda_{\#}-\delta<\lambda<\lambda_{\#}+\delta$, $0<\delta<\infty$, and where $k, g$ and $f$ satisfy conditions which will insure that a solution exists when appropriate initial conditions are specified.

The system $(\mathrm{NH})$ will be considered together with two-point boundary conditions of the form

$$
\begin{gathered}
\alpha(\lambda) y(a, \lambda)-\beta(\lambda) z(a, \lambda)=0, \\
\gamma_{1}(\lambda) y(a, \lambda)-\delta_{1}(\lambda) z(a, \lambda)=\gamma_{2}(\lambda) y(b, \lambda)-\delta_{2}(\lambda) z(b, \lambda),
\end{gathered}
$$

Presented to the Society, March 20, 1975; received by the editors July 24, 1974. $34 \mathrm{C} 20$.

AMS (MOS) subject classifications (1970). Primary 34B25, 34B05; Secondary

Key words and phrases. Nonhomogeneous differential system, polar coordinate transformation, boundary value problem, eigenvalue. 
where $\alpha, \beta, \gamma_{i}, \delta_{i}, i=1,2$, are continuous, real-valued functions on $L$.

This work will extend the results of Whyburn and Etgen to the problem (NH, la, b). This will be accomplished by establishing a polar coordinate transformation for the system (NH).

It is well known that the problem $(\mathrm{NH}, 1 \mathrm{a}, \mathrm{b})$ will have a unique solution for those values of $\lambda$ for which the associated homogeneous problem $(\mathrm{H}, 1 \mathrm{a}, \mathrm{b})$ has only the trivial solution. Further, for those values of $\lambda$ for which $(\mathrm{H}, \mathrm{la}, \mathrm{b})$ has a nontrivial solution, $(\mathrm{NH}, 1 \mathrm{a}, \mathrm{b})$ may have zero or infinitely many solutions. In either case, the homogeneous problem must be solved before solutions of the nonhomogeneous problem can be determined. This seems to be an indirect method of solution.

For those values of $\lambda$ for which $(\mathrm{H}, \mathrm{la}, \mathrm{b})$ has a nontrivial solution, Max Mason [4] gave necessary and sufficient conditions for a nontrivial solution of $(\mathrm{NH}, 1 \mathrm{a}, \mathrm{b})$ to exist. However, these conditions are highly dependent on the fact that the boundary problem be selfadjoint. For the boundary conditions ( $1 \mathrm{a}, \mathrm{b})$, this would imply that $a(\lambda) \delta_{1}(\lambda)-\beta(\lambda) \gamma_{1}(\lambda) \equiv 0$ on $L$.

Assuming that $k(x, \lambda)$ is nonzero on $X L$, the system $(\mathrm{NH})$ can be written

$$
\left(y^{\prime} / k\right)^{\prime}-g y=f,
$$

and the associated homogeneous equation is

$$
\left(u^{\prime} / k\right)^{\prime}-g u=0 .
$$

It is easily verified that the general solution of (3) is given by

$$
\begin{aligned}
y(x, \lambda)= & {\left[c_{1}(\lambda)-\int_{a}^{x} f(t, \lambda) v(t, \lambda) d t\right] u(x, \lambda) } \\
& +\left[c_{2}(\lambda)+\int_{a}^{x} f(t, \lambda) u(t, \lambda) d t\right] v(x, \lambda),
\end{aligned}
$$

where $\{u(x, \lambda), v(x, \lambda)\}$ is a solution basis of (4) such that $\left(u v^{\prime}-v u^{\prime}\right) / k$ $\equiv 1$ on $X L$.

Suppose that $u\left(x, \lambda_{i}\right)$ and $v\left(x, \lambda_{i}\right)$ are linearly independent solutions of $(4,1 \mathrm{a}, \mathrm{b})$, for the value $\lambda=\lambda_{i}$. Mason has shown that a necessary and sufficient condition for the existence of a nontrivial solution of $(3,1 \mathrm{a}, \mathrm{b})$, is that

$$
D\left(\lambda_{i}\right) \int_{a}^{x} f\left(t, \lambda_{i}\right) u\left(t, \lambda_{i}\right) d t=D\left(\lambda_{i}\right) \int_{a}^{x} f\left(t, \lambda_{i}\right) v\left(t, \lambda_{i}\right) d t=0,
$$

where $D\left(\lambda_{i}\right)=a\left(\lambda_{i}\right) \delta_{1}\left(\lambda_{i}\right)-\beta\left(\lambda_{i}\right) \gamma_{1}\left(\lambda_{i}\right)$. Imposing the selfadjointness condition would imply that $D(\lambda) \equiv 0$ on $L$, and the above equations would trivially be satisfied for any linearly independent pair of solutions of (4).

If we suppose instead that $D(\lambda) \neq 0$ on $L$, then the above equations become 


$$
\int_{a}^{b} f\left(t, \lambda_{i}\right) u\left(t, \lambda_{i}\right) d t=\int_{a}^{b} f\left(t, \lambda_{i}\right) v\left(t, \lambda_{i}\right) d t=0
$$

This would imply that $y_{p}\left(b, \lambda_{i}\right)=y_{p}^{\prime}\left(b, \lambda_{i}\right)=0$, where $y_{p}\left(x, \lambda_{i}\right)$ is obtained from (5), by putting $c_{1}=c_{2}=0$ [Theorem 2.10,5]. If it is assumed that for each $\lambda \in L, f(x, \lambda)>0, f^{\prime}(x, \lambda)>0, k^{\prime}(x, \lambda) \leq 0$, and $g^{\prime}(x, \lambda) \geq 0$ on $X$, then $y_{p}\left(x, \lambda_{i}\right)>0$ on $(a, \infty)$, and the boundary problem $(\mathrm{NH}, 1 \mathrm{a}, \mathrm{b})$ could not be solved [Theorem $2.11,5]$. This indicates the need for another method of solution.

The following hypotheses on the coefficients involved in (NH), (1a), and (1b) will be assumed throughout:

$\left(\mathrm{H}_{1}\right)$ For each $x \in X$, each of $k(x, \lambda), g(x, \lambda)$ and $f(x, \lambda)$ is continuous on $L$.

$\left(\mathrm{H}_{2}\right)$ For each $\lambda \in L$, each of $k(x, \lambda), g(x, \lambda)$ and $f(x, \lambda)$ is measurable on $X$.

$\left(\mathrm{H}_{3}\right)$ There exists a Lebesgue integrable function $M(x)$ on $X$ such that $|k(x, \lambda)| \leq M(x),|g(x, \lambda)| \leq M(x)$, and $|f(x, \lambda)| \leq M(x)$ on $X L$.

$\left(\mathrm{H}_{4}\right) k(x, \lambda)>0$ on $X L$.

$\left(\mathrm{H}_{5}\right)$ Each of the functions $\alpha(\lambda), \beta(\lambda), \gamma_{i}(\lambda), \delta_{i}(\lambda), i=1,2$, is continuous on $L$.

$\left(\mathrm{H}_{6}\right) a^{2}(\lambda)+\beta^{2}(\lambda)>0, \gamma_{i}^{2}(\lambda)+\delta_{i}^{2}(\lambda)>0, i=1,2$, on $L$. In particular, without loss of generality, we assume $\alpha^{2}(\lambda)+\beta^{2}(\lambda) \equiv 1 \equiv \gamma_{1}^{2}(\lambda)+\delta_{1}^{2}(\lambda)$ on $L$. $\left(\mathrm{H}_{7}\right) \beta(\lambda)>0, \gamma_{2}(\lambda) \beta(\lambda)-\delta_{2}(\lambda) \alpha(\lambda) \neq 0$ on $L$.

2. Preliminary results. We establish the existence of values of $\lambda$ on $L$ for which there corresponds a nontrivial solution of $(\mathrm{NH})$ satisfying ( $1 \mathrm{a}, \mathrm{b})$. Such values of $\lambda$ are called eigenvalues of the boundary problem. By a nontrivial solution of $(\mathrm{NH})$ we mean a solution pair $\{y(x, \lambda), z(x, \lambda)\}$ of (NH) such that $y^{2}(x, \lambda)+z^{2}(x, \lambda) \neq 0$ on $X L$. This definition differs from that for a homogeneous system because it is possible to have values of $x$ such that $y^{2}(x, \lambda)+z^{2}(x, \lambda)=0$, i.e. "double zeros" can exist for solutions of $(\mathrm{NH})$.

It is readily seen that if hypotheses $\left(\mathrm{H}_{1}\right)-\left(\mathrm{H}_{3}\right)$ are satisfied and if $\{y(x, \lambda), z(x, \lambda)\}$ is any solution pair of $(\mathrm{NH})$ such that $y^{2}(x, \lambda)+z^{2}(x, \lambda)$ $>0$ on $X L$, then there exists a pair of functions $\rho(x, \lambda), \theta(x, \lambda)$ with the property that

$$
y(x, \lambda)=\rho(x, \lambda) \sin \theta(x, \lambda), \quad z(x, \lambda)=\rho(x, \lambda) \cos \theta(x, \lambda) .
$$

Furthermore, $\rho(x, \lambda)$ and $\theta(x, \lambda)$ satisfy the differential equations

$$
\begin{aligned}
& \text { (a) } \rho^{\prime}=\rho(k+g) \sin \theta \cos \theta+f \cos \theta, \\
& \text { (b) } \theta^{\prime}=k \cos ^{2} \theta-g \sin ^{2} \theta-(f \sin \theta) / \rho \text {, }
\end{aligned}
$$

with the initial conditions $\rho(a)>0 ; 0 \leq \theta(a) \leq 2 \pi$. 
It is required that $y^{2}(x, \lambda)+z^{2}(x, \lambda)>0$ on $X L$ to insure that $\rho^{\prime}(x, \lambda)$ and $\theta^{\prime}(x, \lambda)$ are defined on $X L$. The following theorem provides conditions under which these functions are well defined [6].

Theorem 1. Suppose that $\rho(a, \lambda)+1>\exp \int_{a}^{b} M(s) d s$ on $L$, where $\rho(x, \lambda)$ is defined by (7), and $M(x)$ is the Lebesgue integrable bound of the coefficients. Then $\rho(x, \lambda)>0$ and consequently, $y^{2}(x, \lambda)+z^{2}(x, \lambda)>0$ on $X L$.

Proof. Solving the first order, linear equation (7a), one obtains

(8) $\rho(x, \lambda)=\exp w(x, \lambda)\left\{\rho(a, \lambda)+\int_{a}^{x}[f(t, \lambda) \cos \theta(t, \lambda)] \exp (-w(t, \lambda)) d t\right\}$, where

$$
w(x, \lambda)=\int_{a}^{x}[k(t, \lambda)+g(t, \lambda)] \sin \theta(t, \lambda) \cos \theta(t, \lambda) d t .
$$

It is clear that $\rho(x, \lambda)=0$ only if the expression inside the braces is zero. Now

$$
\left|\int_{a}^{x}(f \cos \theta) \exp (-w) d t\right| \leq \int_{a}^{x} M(t) \exp \int_{a}^{t} M(s) d s d t=\exp \int_{a}^{x} M(t) d t-1 .
$$

Hence if $\rho(a, \lambda)+1>\exp \int_{a}^{b} M(s) d s$, then $\rho(x, \lambda)>0$ on $X L$ 。

Hypotheses $\left(\mathrm{H}_{1}\right)-\left(\mathrm{H}_{3}\right)$ allow application of fundamental existence theorems (see e.g. [1, Chapter 2]) for differential systems to obtain the existence of a solution pair $\{y(x, \lambda), z(x, \lambda)\}$ of $(\mathrm{NH})$ on $X L$ such that

$$
y(a, \lambda) \equiv \beta(\lambda) ; \quad z(a, \lambda) \equiv \alpha(\lambda)
$$

on $L$. Further, $\{y(x, \lambda), z(x, \lambda)\}$ has the polar coordinate representation (6), with $\{\rho(x, \lambda), \theta(x, \lambda)\}$ solutions of (7) with the initial conditions

$$
\rho(a, \lambda) \equiv 1, \quad \sin \theta(a, \lambda) \equiv \beta(\lambda), \quad \cos \theta(a, \lambda) \equiv \alpha(\lambda), \quad 0<\theta(a, \lambda)<2 \pi .
$$

Since $\beta(\lambda)>0$, we may assume $0<\theta(a, \lambda)<\pi$.

We now define the functions

$$
\begin{aligned}
& c(x, \lambda)=\gamma_{2}(\lambda) y(x, \lambda)-\delta_{2}(\lambda) z(x, \lambda), \\
& d(x, \lambda)=\gamma_{2}(\lambda) z(x, \lambda)+\delta_{2}(\lambda) y(x, \lambda) .
\end{aligned}
$$

Assuming the hypothesis of Theorem 1 , it follows that $c^{2}(x, \lambda)+$ $d^{2}(x, \lambda)>0$ on $X L$ and $z(x, \lambda)-i y(x, \lambda)$ is nonzero on $X L$. Hence the complex-valued functions

$$
\begin{aligned}
& \theta(x, \lambda)=[z(x, \lambda)+i y(x, \lambda)] /[z(x, \lambda)-i y(x, \lambda)], \\
& \psi(x, \lambda)=[d(x, \lambda)+i c(x, \lambda)] /[d(x, \lambda)-i c(x, \lambda)]
\end{aligned}
$$

are well defined on $X L$.

Theorem 2. The complex-valued functions $\phi(x, \lambda)$ and $\psi(x, \lambda)$ have the following properties on $X$ for each $\lambda \in L$ : 
(i) $|\phi(x, \lambda)|=|\psi(x, \lambda)|=1$.

(ii) Both $\phi(x, \lambda)$ and $\psi(x, \lambda)$ satisfy the first order differential equation $d \phi / d x=2 i \phi j(x, \lambda)$, where

$$
j(x, \lambda)=\left(k z^{2}-g y^{2}-f y\right) /\left(y^{2}+z^{2}\right)=k \cos ^{2} \theta-g \sin ^{2} \theta-(f \sin \theta) / \rho,
$$

(iii) $\phi(x, \lambda)=+1$ if and only if $y(x, \lambda)=0, \phi(x, \lambda)=-1$ if and only if $z(x, \lambda)=0, \psi(x, \lambda)=+1$ if and only if $c(x, \lambda)=0$, and $\psi(x, \lambda)=-1$ if and only if $d(x, \lambda)=0$.

(iv) Let $\mu(x, \lambda)=\arg \phi(x, \lambda)$ and $\zeta(x, \lambda)=\arg \psi(x, \lambda)$, where we assume $0 \leq \mu\left(a, \lambda_{\#}\right), \zeta\left(a, \lambda_{\#}\right)<2 \pi$, and that $\mu(x, \lambda)$ and $\zeta(x, \lambda)$ are continued as continuous functions on $X L$. Then

$$
2 \int_{a}^{x} j(t, \lambda) d t=\zeta(x, \lambda)-\zeta(a, \lambda)=\mu(x, \lambda)-\mu(a, \lambda) .
$$

(v) $\phi(x, \lambda)$ moves monotonically and positively on the unit circle at +1 .

Let $\{y(x, \lambda), z(x, \lambda)\}$ be a solution pair of (NH) satisfying (10). Then boundary condition (1a) is satisfied. Using the polar form of $y$ and $z$ and the definitions of $c(x, \lambda)$ and $d(x, \lambda)$, boundary condition (1b) can be written

(15) $\sin \left[\theta(a, \lambda)-\tau_{1}(\lambda)\right]=c(b, \lambda)=\rho(b, \lambda)\left[\gamma_{2}^{2}(\lambda)+\gamma_{2}^{2}(\lambda)\right]^{1 / 2} \sin \left[\theta(b, \lambda)-\tau_{2}(\lambda)\right]$, where

$$
\begin{gathered}
\sin \tau_{i}(\lambda)=\delta_{i}(\lambda) /\left[\gamma_{i}^{2}(\lambda)+\delta_{i}^{2}(\lambda)\right]^{1 / 2}, \\
\cos \tau_{i}(\lambda)=\gamma_{i}(\lambda) /\left[\gamma_{i}^{2}(\lambda)+\delta_{i}^{2}(\lambda)\right]^{1 / 2}, \quad i=1,2 .
\end{gathered}
$$

By hypotheses $\left(\mathrm{H}_{7}\right), c(a, \lambda)=\gamma_{2}(\lambda) \beta(\lambda)-\delta_{2}(\lambda) \alpha(\lambda) \neq 0$ on $L$, which implies $0<\zeta(a, \lambda)<2 \pi$ on $L$. Also from $\left(\mathrm{H}_{7}\right)$, we have that $0<\mu(a, \lambda)<$ $2 \pi$ on $L$. Therefore, the following inequalities hold for each $\lambda$ :

$$
2 \int_{a}^{b} j(t, \lambda)<\mu(b, \lambda), \quad \zeta(b, \lambda)<2 \int_{a}^{b} j(t, \lambda) d t+2 \pi .
$$

3. Existence of eigenvalues. We can now apply the tools developed in the previous section to determine the existence of eigenvalues for the nonhomogeneous boundary problem $(\mathrm{NH}, 1 \mathrm{a}, \mathrm{b})$.

Theorem 3. Let $\{y(x, \lambda), z(x, \lambda)\}$ be the solution of (NH) given by (10). Assume the hypothesis of Theorem 1, and define

$$
R(\lambda)=2 \int_{a}^{b} j(t, \lambda) d t
$$

$(j(t, \lambda)$ defined by (13)). Then $R(\lambda)>-2 \pi$ on $L$. Let $m \geq-1$ be the least integer such that inf $R(\lambda)<(2 m+1)$ on $L$, and let $n$ be an integer such that 
sup $R(\lambda)>(2 n+1)$ on L. Suppose $n \geq m+1$. If any of the following conditions hold on $L$ :

$$
\begin{array}{r}
\int_{a}^{b} f(t, \lambda) \cos \theta(t, \lambda) \exp [-w(t, \lambda)] d t \geq 0, \\
\int_{a}^{b}[k(t, \lambda)+g(t, \lambda)] \sin \theta(t, \lambda) \cos \theta(t, \lambda) d t \geq 0,
\end{array}
$$

and $\gamma_{2}^{2}(\lambda)+\delta_{2}^{2}(\lambda) \geq 1$

$$
\begin{aligned}
\int_{a}^{b} w(t, \lambda) d t \geq-\{ & \ln \left[\gamma_{2}^{2}(\lambda)+\delta_{2}^{2}(\lambda)\right]^{1 / 2} \\
& \left.+\ln \left[1+\int_{a}^{b} f(t, \lambda) \cos \theta(t, \lambda) \exp [-w(t, \lambda)] d t\right]\right\}
\end{aligned}
$$

$(w(t, \lambda)$ defined by $(9) ; \theta(t, \lambda)$ defined by (7))

(iii) $4 \int_{a}^{b} M(s) d s \leq \ln \left[\gamma_{2}^{2}(\lambda)+\delta_{2}^{2}(\lambda)\right]$, where $M(x)$ is the Lebesgue integrable bound of the functions $k(x, \lambda), g(x, \lambda)$, and $f(x, \lambda)$, then there exists at least $p, p=n-m$, nonempty sets of eigenvalues $T_{0}, T_{1}, \ldots, T_{p-1}$ for $(\mathrm{NH}, \mathrm{la}, \mathrm{b})$.

Proof. Let the solution pair $\{y(x, \lambda), z(x, \lambda)\}$ be defined by (10). Then this is a solution of (NH, la). Let $\phi(x, \lambda), \psi(x, \lambda), c(x, \lambda), d(x, \lambda)$ be defined as above.

Since many of the techniques used in this proof are similar to those used in [2], the reader is referred to [2] for many of the details.

If $m$ and $n$ are integers with the properties described in the hypotheses, then there exist two distinct values of $\lambda$, say $\bar{\lambda}$ and $\lambda^{*}, \lambda^{*}<\bar{\lambda}$, such that $R\left(\lambda^{*}\right)=(2 m+1) \pi$ and $R(\bar{\lambda})=(2 n+1) \pi$. From $(16)$, we have $\zeta\left(b, \lambda^{*}\right)<$ $(2 m+3) \pi$ and $\zeta(b, \bar{\lambda})>(2 n+1) \pi$. Therefore, there exist $p$ values of $\lambda, \lambda_{0}$, $\lambda_{1}, \ldots, \lambda_{p-1}$ on $\left(\lambda^{*}, \bar{\lambda}\right)$ such that $\zeta\left(b, \lambda_{j}\right)=[2(m+j)+3] \pi$. We may assume $\lambda_{0}<\lambda_{1}<\cdots<\lambda_{p-1}$ from the continuity of $\zeta(b, \lambda)$. Thus $\psi\left(b, \lambda_{j}\right)=-1$ for $j=0,1, \ldots, p-1$. Expressing $c(x, \lambda)$ and $d(x, \lambda)$ in polar form, we have

$$
\begin{aligned}
& c(b, \lambda)=\rho(b, \lambda)\left[\gamma_{2}^{2}(\lambda)+\delta_{2}^{2}(\lambda)\right]^{1 / 2} \sin \left[\theta(b, \lambda)-\tau_{2}(\lambda)\right], \\
& d(b, \lambda)=\rho(b, \lambda)\left[\gamma_{2}^{2}(\lambda)+\delta_{2}^{2}(\lambda)\right]^{1 / 2} \cos \left[\theta(b, \lambda)-\tau_{2}(\lambda)\right] .
\end{aligned}
$$

Thus for each $j, d\left(b, \lambda_{j}\right)=0$ implies $\sin \left[\theta\left(b, \lambda_{j}\right)-\tau_{2}\left(\lambda_{j}\right)\right]= \pm 1$, and as $\lambda$ increases from $\lambda_{j}$ to $\lambda_{j+1}, \sin \left[\theta(b, \lambda)-\tau_{2}(\lambda)\right]$ changes continuously in value from +1 to -1 or -1 to +1 .

Consider equation (15). We know that $\left|\sin \left[\theta(a, \lambda)-\tau_{1}(\lambda)\right]\right| \leq 1$ on $L$. Let $G(\lambda)=\rho(b, \lambda)\left[\gamma_{2}^{2}(\lambda)+\delta_{2}^{2}(\lambda)\right]^{1 / 2}$. From the preceding paragraph, we have that $c(b, \lambda)$ changes continuously from $+G\left(\lambda_{j}\right)$ to $-G\left(\lambda_{j+1}\right)$ or $-G\left(\lambda_{j}\right)$ to $+G\left(\lambda_{j+1}\right)$, as $\lambda$ increases from $\lambda_{j}$ to $\lambda_{j+1}$, for each $j$. Hence, if we can 
show that $G(\lambda) \geq 1$ on $L$, then for each $j$, there must exist a value of $\lambda$ in $\left[\lambda_{j}, \lambda_{j+1}\right]$ with the property that (1b), or (15) is satisfied.

From $\left(\mathrm{H}_{6}\right)$ and $(10), \rho(a, \lambda) \equiv 1$ on $L$, so from (8) and (9),

$$
\rho(b, \lambda)=\exp w(b, \lambda)\left(1+\int_{a}^{b} f(t, \lambda) \cos \theta(t, \lambda) \exp [-w(t, \lambda)] d t\right) .
$$

Under condition (i), exp $w(b, \lambda) \geq 1$,

$$
1+\int_{a}^{b} f(t, \lambda) \cos \theta(t, \lambda) \exp [-w(t, \lambda)] d t \geq 1,
$$

and, consequently, $\rho(b, \lambda) \geq 1$ on $L$. Assuming also that $\gamma_{2}^{2}(\lambda)+\delta_{2}^{2}(\lambda) \geq 1$ on $L$ yields the desired result.

The implication of condition (ii) is that

$$
\begin{aligned}
& \exp \int_{a}^{b} w(t, \lambda) d t \\
& \quad \geq 1 /\left(1+\int_{a}^{b} f(t, \lambda) \cos \theta(t, \lambda) \exp [-w(t, \lambda)] d t\right)\left[\gamma_{2}^{2}(\lambda)+\delta_{2}^{2}(\lambda)\right]^{1 / 2}
\end{aligned}
$$

on $L$, or that $G(\lambda) \geq 1$ on $L$.

For condition (iii), we see that

$$
|w(b, \lambda)| \leq \frac{1}{2} \int_{a}^{b}|k(t, \lambda)+g(t, \lambda)| \cdot|\sin 2 \theta(t, \lambda)| d t \leq \int_{a}^{b} M(t) d t .
$$

Therefore,

$$
\rho(b, \lambda) \geq\left(1-\int_{a}^{b} M(t) \exp \left[-\int_{a}^{t} M(s) d s\right] d t\right) \exp \left[-\int_{a}^{b} M(t) d t\right]
$$

or $\rho(b, \lambda) \geq \exp \left[-2 \int_{a}^{b} M(t) d t\right]$. So, if (iii) holds, then

$$
\begin{aligned}
& -2 \int_{a}^{b} M(s) d s \geq \ln \left[\gamma_{2}^{2}(\lambda)+\delta_{2}^{2}(\lambda)\right]^{-1 / 2}, \\
& {\left[\gamma_{2}^{2}(\lambda)+\delta_{2}^{2}(\lambda)\right]^{1 / 2} \exp \left[-2 \int_{a}^{b} M(s) d s\right] \geq 1,}
\end{aligned}
$$

and again $G(\lambda) \geq 1$ on $L$.

Letting $T_{j}$ be the set of all eigenvalues on $\left[\lambda_{j}, \lambda_{j+1}\right], j=0,1, \ldots$, $p-1$, the proof is completed.

Corollary 1. Under the hypotheses of Theorem 3, if the integer $n$ can be chosen arbitrarily large, then there exist infinitely many nonempty sets of eigenvalues $T_{0}, T_{1}, T_{2}, \ldots$, for $(\mathrm{NH}, 1 \mathrm{a}, \mathrm{b})$.

Corollary 2. Under the hypotheses of Theorem 3, there exist $p$ nonempty sets of eigenvalues $J_{0}, J_{1}, \ldots, J_{p-1}$ for $(\mathrm{NH}, 1 \mathrm{a}, \mathrm{b})$, so that if $\sigma_{j} \in J_{j}, j=0,1, \ldots, p-1$, then $\zeta\left(b, \sigma_{j}\right) \geq[2(m+j)+3] \pi$. Furthermore, if $j \geq 1$, then the corresponding solution $y\left(x, \sigma_{j}\right)$ has at least $j-1$ zeros on $X$. 
In proving Theorem 3, we used the hypothesis that $\alpha(\lambda) \gamma_{2}(\lambda)-\beta(\lambda) \delta_{2}(\lambda)$ $\neq 0$ on $L$, to show that $0<\zeta(a, \lambda)<2 \pi$ on $L$, and, consequently, $R(\lambda)<$ $\zeta(b, \lambda)<R(\lambda)+2 \pi$. If we assume instead that $a(\lambda) \gamma_{2}(\lambda)-\beta(\lambda) \delta_{2}(\lambda) \equiv 0$ on $L$, then $\zeta(a, \lambda) \equiv 0$ and $R(\lambda)=\zeta(b, \lambda)$ on $L$. Theorem 3 then follows with simple modifications in the proof.

If we assume the selfadjointness condition, i.e. if $\alpha(\lambda) \delta_{1}(\lambda)-\beta(\lambda) \gamma_{1}(\lambda)$ $\equiv 0$ on $L$, then the boundary condition (1b) becomes $\gamma_{2}(\lambda) y(b, \lambda)-$ $\delta_{2}(\lambda) z(b, \lambda)=0$, and equation (15) is

$$
\rho(b, \lambda)\left[\gamma_{2}^{2}(\lambda)+\delta_{2}^{2}(\lambda)\right]^{1 / 2} \sin \left[\theta(b, \lambda)-\tau_{2}(\lambda)\right]=0 .
$$

Consequently, the eigenvalues for this problem will be those values of $\lambda$ such that $\left[\theta(b, \lambda)-\tau_{2}(\lambda)\right] \equiv 0(\bmod \pi)$.

We then have

Theorem 4. Let $\{y(x, \lambda), z(x, \lambda)\}$ be the solution of (NH) given by (10). Suppose that $a(\lambda) \delta_{1}(\lambda)-\beta(\lambda) \gamma_{1}(\lambda) \equiv 0$ on L. Define $R(\lambda)$ as in Theorem 3 . Then $R(\lambda)>-2 \pi$ on $L$. Let $m \geq 0$ be the least integer such that inf $R(\lambda)$ $<2 m \pi$ on $L$, and let $n$ be an integer such that sup $R(\lambda)>2 n \pi$ on L. Suppose $n \geq m+1$. Then there exist at least $p, p=n-m$, nonempty sets of eigenvalues $T_{0}, T_{1}, \ldots, T_{p-1}$ for $(\mathrm{NH}, 1 \mathrm{a}, \mathrm{b})$.

Corollaries analogous to those of Theorem 3 are valid for Theorem 4 .

\section{BIBLIOGR APHY}

1. E. A. Coddington and N. Levinson, Theory of ordinary differential equations, McGraw-Hill, New York, 1955. MR 16, 1022.

2. G. J. Etgen and S. C. Tefteller, A two-point boundary problem for nonlinear second order differential systems, SIAM J. Math. Anal. 2 (1971), 64-71. MR 44 \#5544.

3. - Second order differential equations with general boundary conditions, SIAM J. Math. Anal. 3 (1972), 512-519. MR 47 \# 538.

4. Max Mason, On the boundary value problems of linear ordinary differential equations of second order, Trans. Amer. Math. Soc. 7 (1906), 337-360.

5. S. C. Tefteller, Oscillation of second order nonhomogeneous linear differential equations, SIAM J. Appl. Math. (to appear).

6. - A polar coordinate transformation for nonhomogeneous differential systems (submitted).

7. W. M. Whyburn, Existence and oscillation theorems for non-linear systems of the second order, Trans. Amer. Math. Soc. 30 (1928), 848-854.

8. - A nonlinear boundary value problem for second order differential systems, Pacific J. Math. 5 (1955), 147-160. MR 16, 1027.

9. A note on a non-self-adjoint differential system of the second order, J. Elisha Mitchell Sci. Soc. 69 (1953), 116-118.

DEPARTMENT OF MATHEMATICS, UNIVERSITY OF ALABAMA AT BIRMINGHAM, BIRMINGHAM, ALABAMA 35294 